Portland State University

PDXScholar

8-18-2020

\title{
Numerical Model of a Radio Frequency lon Source for Fusion Plasma Using Particle-In-Cell and Finite Difference Time Domain
}

Augustin L. Griswold

Portland State University

Follow this and additional works at: https://pdxscholar.library.pdx.edu/honorstheses

Part of the Numerical Analysis and Scientific Computing Commons, and the Plasma and Beam Physics Commons

Let us know how access to this document benefits you.

\section{Recommended Citation}

Griswold, Augustin L., "Numerical Model of a Radio Frequency lon Source for Fusion Plasma Using Particle-In-Cell and Finite Difference Time Domain" (2020). University Honors Theses. Paper 932. https://doi.org/10.15760/honors.955

This Thesis is brought to you for free and open access. It has been accepted for inclusion in University Honors Theses by an authorized administrator of PDXScholar. Please contact us if we can make this document more accessible: pdxscholar@pdx.edu. 
Numerical Model of a Radio Frequency Ion Source for Fusion Plasma Using ParticleIn-Cell and Finite Difference Time Domain

$$
\text { by }
$$

Augustin L. Griswold

An undergraduate honors thesis submitted in partial fulfillment of the requirements for the degree of

Bachelor of Science

in

University Honors

and

Physics

Thesis Adviser

Erik Sánchez

Portland State University 


\title{
Numerical Model of a Radio Frequency Ion Source for Fusion Plasma Using Particle-In-Cell and Finite Difference Time Domain
}

\author{
Augustin Griswold \\ Department of Physics, Portland State University. \\ P.O. Box 751, Portland, Oregon 97207-0751
}

(Dated: August 17, 2020)

\begin{abstract}
Radio frequency (RF) plasma sources are common tool for application and study, and of particular interest for inertial electrostatic (IEC) fusion. Computational analysis is often carried out using particle in cell (PIC) methods or finite difference time domain (FDTD). However, a more holistic analysis is necessary as the particle distribution is highly dependant on the fields created by the plasma source. Herein, an analysis of a particular planar RF electrode with deuterium gas is provided which covers the fields and the particle behaviour using first FDTD then PIC. Further applications are discussed as well as further directions for this study.
\end{abstract}

\section{INTRODUCTION}

In inertial electrostatic confinement fusion (IEC), high ion currents are desirable to achieve high fusion rates. This is given by Miley as a modified version of the Lawson Criterion: or reactions occurring only with beam-beam interactions, the fusion rate, $f$, is

$$
f=n_{d}^{2} \overline{v \sigma(T)}
$$

with $n_{d}$ being the density of fuel (deuterium in this case) and $\overline{v \sigma(T)}$ is the cross sectional area of the reaction average over all the velocities of the species. Specifically, this is the beam-beam fusion rate per volume. Compared to the background-beam fusion rate per volume,

$$
f=n_{b k} n_{d} \overline{v \sigma(T)},
$$

where $n_{b k}$ is the density of the background species. IEC devices are designed with a semitransparent mesh held at high voltage within a vacuum chamber. The is held in by a stalk and ions are fed into it via some ion source. As it is the novel aspect of an IEC, the grid has been the focus of research. Similarly, the stalk requires a thin, and resilient design as it is likely to face ion implantation[1] and breakdown, so it too has been a topic of research[2]. Other than adding more ion sources to an IEC, the ion source does not garner much attention even though a few studies have seen improvements coming from choice of ion source [3]. In fact, the general development of ion sources aids many more fields than just IEC fusion. The devices themselves are embedded into the private sector and see novel applications (such as the Plasma Focused Ion Beam [4]) today).

While the physics within the various types of plasma sources are well understood, the analysis and optimization of these devices is somewhat limited. In most cases PIC methods[5][6][7] or FDTD[8], rarely both[9]. However, given the ability of modern computing, more thorough and rigorous numerical solutions can be reached by mixing the three methods. An analysis of an RF ion source is provided here as a case study to display the process.

\section{IEC FUSION}

Nuclear fusion research has been carried out since the 1940s, but, as of yet, has not reached break-even as shown by the net power equation $(\mathrm{P})$ :

$$
P=\eta\left(P_{f}-P_{c}-P_{r}\right),
$$

where $\eta$ is the efficiency, $P_{f}$ is energy output by fusion processes, $P_{c}$ is the power lost to conduction and $P_{r}$ is the power lost to radiative processes like Bremstrahlung radiation. This equation shows the overall goal of fusion, but is nebulous as to what the dependant variables. Instead, the more useful relation is fusion rate[10]. Trivially modified versions of this were shown in equations 1 and 2 , but for two species of gas $n_{1}$ and $n_{2}$, the fusion rate per volume is given by

$$
f=n_{1} n_{2} \overline{v \sigma(T)} .
$$

The goal of IEC fusion is to hold deuterium ions within a grid (which is used as a spherically convergent ion focus) in order to achieve high density regions in the hope of increasing the fusion yield. The major components of IECs were brought up before, but for the sake of completeness: the high voltage is held in a vacuum chamber by a stalk and ions enter the system via some ion source.

The grid is the aspect of the device which has received the most attention. Most studied s have been spherical in shape with either geodesic -lines or semi-Cartesian lines[11] (though often these differences are ornamental as the performance in terms of symmetry is saturated). Due to the original IEC design[12], the majority of fusors have used spherical geometry[2], however, there are a few notable studies which have looked at cylindrical or semispherical designs. For cylindrical designs, the goal is to create a dense core region, which is particularly useful for neutron sources. These designs come in two types: hollow cathode (C-type) and 2D ded (cylindrical IEC. Either design is not suitable as a fusion source due to the scaling: as the z-axis increases, so does the power consumption. It is however ideal for beam-beam fusion[2]. However, since they lack the third axis of the spherical system, they show lower neutron rates overall[13]. 
For systems running a multi-well[14] configuration a desirable trait in a grid, regardless of the geometry, is electron emission. Since the system operates at high temperatures and fields, thermionic emission is the best way to do so. The high temperature results in high emission, while the fields lower the work function. This improves ion space charge within the grid and allows for extra ion production for neutrals which may stream into the chamber. However, these are all negatives for systems running a single well system. The power input $P_{\text {ext }}$ is defined by

$$
P_{e x t}=\left(i_{i}+i_{e i}+i_{e o}\right) V
$$

where $i_{i}$ is the ion current, $i_{e i}$ is the electron current from the inner surface of the grid and $i_{e o}$ is the electron current from the outer surface of the grid. Note that as the electron currents increase, the ion currents lower, resulting in a lower fusion yield. As such, an important criteria for a single well system must support high ion bombardment and have a high work function to lower thermionic emission, and a high melting point. It must also be resistant to sputtering, as the grid material could coat the stalk, causing pathways for dielectric breakdown, or, under continued use, a short circuit. It was found that a W-Re (25:75) alloy met the criteria above, and was easy to manufacture[15].

\section{FINITE DIFFERENCE METHOD}

The Finite Difference Method (FDM) is a particularly useful tool in computational physics as it provides numerical solutions to ordinary differential equations and nonlinear partial differential equations by applying difference equations to derivatives. The method is employed by many fields, but is of particular interest in computational electrodynamics as it can be useful in solving Maxwell's equations for complicated systems.

\section{A. Finite Difference Time Domain}

FDTD is a method in which the electric and magnetic fields of a system are solved suing a staggered system (a Yee lattice, named after Kane Yee and shown in FIG. 1) in which each point on the must satisfy Maxwell's equations. For non-dispersive media, fields are calculated via

$$
\begin{gathered}
\frac{B_{z}^{n+1 / 2}\left(i, j+\frac{1}{2}, k+\frac{1}{2}\right)-B_{z}^{n-1 / 2}\left(i, j+\frac{1}{2}, k+\frac{1}{2}\right)}{\Delta t}= \\
\frac{E_{y}^{n}\left(i, j+\frac{1}{2}, k+1\right)-E_{y}^{n}\left(i, j+\frac{1}{2}, k\right)}{\Delta z}- \\
\frac{E_{z}^{n}\left(i, j+1, k+\frac{1}{2}\right)-E_{z}^{n-1 / 2}\left(i, j, k+\frac{1}{2}\right)}{\Delta y},
\end{gathered}
$$

where $E$ and $B$ are the electric and magnetic field and $(i, j, k)$ denotes a spatial location within the calculation volume. Similarly, an equation can be constructed for $\vec{D}$ and $\vec{H}$ which include an additional term for $\vec{J}[16]$. The size $(i \Delta x, j \Delta y, k \Delta z)$ must be small enough such that the change in the field from cell to cell is small. Commonly, the Courant factor $S$ is used for cell sizing and the time step size :

$$
\frac{c \Delta t}{\Delta x}=S
$$

where $c$ is the velocity of light and $\Delta t$ is the time difference. Stability itself has been an area of research resulting in a few different metric for stability [17]. The Courant factor is also generalized for other PDE solvers, but the consequence here is that for electrodynamics simulations the wavelengths employed must be much larger than the cell size and the time step must be significantly small such that the change within a single cell is small[18]. The method itself is fairly intuitive, accurate, and robust, giving it a lot of appeal. It has been adapted to provide a diverse set of inputs in terms of materials, cell structure and excitation. More modern adaptions include complex dispersive media [19]. The time-domain solutions allows for broad band excitation or single frequency response.

FDTD has seen almost constant innovation[20] since its introduction. Within the first ten years of Yee's original paper, sinusoidal steady state simulations in three dimensions were implemented [21]. The first, absorbing boundary condition was demonstrated in 1981 [22], with an improved version coming out in 1984[23]. Around 1990, frequency dependant dielectrics were demonstrated for FDTD[24][25][26]. 1994 saw the integration of SPICE (Simulation Program with Integrated Circuit Emphasis) circuits[27] and the implementation of perfectly matched

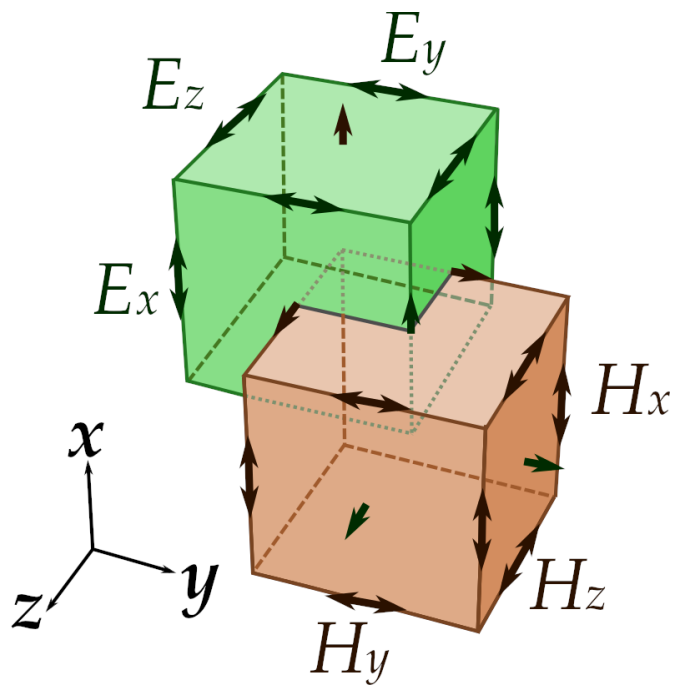

FIG. 1. The characteristic Yee lattice showing the offset $E$ and $B$ fields. Figure was originally created by F. Dominec (Creative Commons). 
layers (PML)[28]. Over time, FDTD has become an incredibly robust method, and despite that, it remains fairly simple.

\section{PARTICLE IN CELL}

PIC is a common tool used to model low density plasma. The process works by solving the Vlasov equation, which solves for distribution function a six dimensional of $\vec{r}, \dot{\vec{r}}=\vec{v}$ and time. The equation ignores collisions, which is applicable in plasma[29] and is written as:

$$
\frac{d}{d t} f(\vec{r}, \vec{v}, t)+v \cdot \nabla_{\vec{r}} f+\frac{q}{m}(\vec{E}+\vec{v} \times \vec{B}) \cdot \nabla_{\vec{v}} f=0,
$$

where $f$ is the distribution function and $\frac{d f}{d t}$ is its total time derivative. This equation demonstrates that along an orbit the particle density stays constant. Looking at particles in the 6 -dimensional space $\vec{r}, \vec{v}$ simplifies the system such that all particles at the same coordinates have the same velocity.

Macro-particles, comprised of many particles, are defined to deal with the distribution function statistically. Considering the one-dimensional case, a cell at $r, v$ will hold a number of macro-particles. They have a velocity, allowing them to move across the $r$ direction in accordance to the polarity of $v$. When particles are affected by a field, they will change their velocity, adjusting their phase-space location. Similarly, when two particles enter a cell, and the conditions for collision are met, there is a probability of them colliding as determined by $\sigma$, the reaction cross section. A portion proportionate to that probability will interact, and particles will have new velocities, or masses in the case of reactions, moving them in the $v$ direction.

\section{METHODS}

The device itself was modelled via Autodesk Inventor. This model included the FR-4 board with the copper electrode embedded, the quartz cup and the poly-carbonate cylindrical shell. For the FDTD, two solutions were considered. First, MEEP (MIT Electromagnetic Equation Propagation)[30] was used in the 2D cylindrical mode (the documentation on the $3 \mathrm{D} \mathrm{C}++$ interface is minimal) using an approximation of the electrode as current loops. While the project was functional, and showed the characteristics one would expect from a set of current loops, it lacked the resonance which is shown by unique geometrical electrodes. Furthermore, while MEEP has been used to analyze plasmas The project was then moved to REMCOM $^{T M}$ 6. While REMCOM ${ }^{T M}$ lacks the efficiency of MEEP, it is useful for providing fully 3D results due to its graphic user interface. It also provides adaptive meshing allowing for fine tuning around parts of the mesh which require high resolution. This allows for the regions of either high detail or of high interest. As a result, the computational cell is defined via meshes shown by Fig 2. Overall, this led to a $648 \times 648 \times 121$ volume, or about 50 million cells.

Via REMCOM ${ }^{T M}$ 's component system, which uses circuits to emulate voltages (either AC or DC) and current densities on an object, the driving circuit for this particular device was emulated running at $27.1 \mathrm{MHz}$. Due to the constraints of FDTD, the first wave is not a true sine wave at the desired frequency. Instead, it ramps up to one in such a way that the Courant factor is still satisfied. As such, the transients introduced by this must be allowed to die out. The program was run for 10 periods (around 2 million iterations) in order to allow this to happen, with the last period being recorded. FIG. 3 shows an example of the fields created via this simulation. The data was recorded every 1000 iterations to reduce the size of the overall data. As a result the data discretized over 384 points ranging from the beginning and end of the period.

While the advanced meshing options of REMCOM ${ }^{T M}$ are advantageous in lowering the total run time of the FDTD simulation, it increases the difficulty of rebuilding the for the PIC simulation. For this task, a program was written in $\mathrm{C}++$ to take in the field and geometry data at each of the REMCOM ${ }^{T M}$ cell locations and interpolate them at the cell locations in the PIC. This involved turning the field files into a class of their own in terms of the header and the fields (E, B, and $\mathrm{J}$ ) at all points within the REMCOM ${ }^{T M}$ computation volume. At run time, the program picked valid time steps, grabbed the file data, and found the valid $\mathrm{REMCOM}^{T M}$ cells surrounding each PIC location. Using four REMCOM ${ }^{T M}$ cells and the PIC location in between, the field data was interpolated using bilinear interpolation to find the field values[18]. Let $\left(x_{0}, y_{0}\right)$ and $\left(x_{1}, y_{1}\right)$ be points forming a of field values $f\left(x_{m}, y_{n}\right)$ and let $(x, y)$ be a point of interest. To find $f(x, y)$ first $f(x, y 0)$ and $f(x, y 1)$ must be

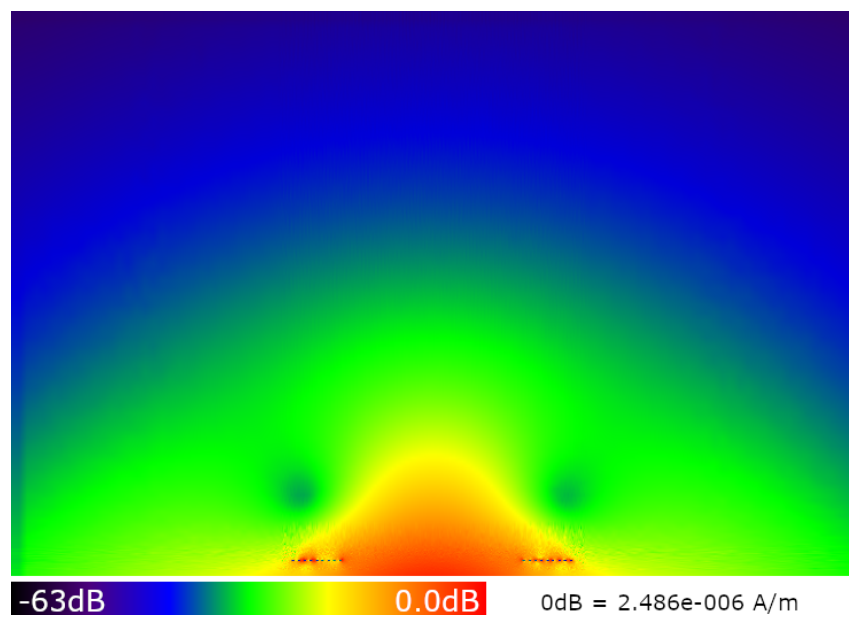

FIG. 2. A rendering of the resulting $\mathrm{H}$ Field at iteration 1830857 of the resonator. 

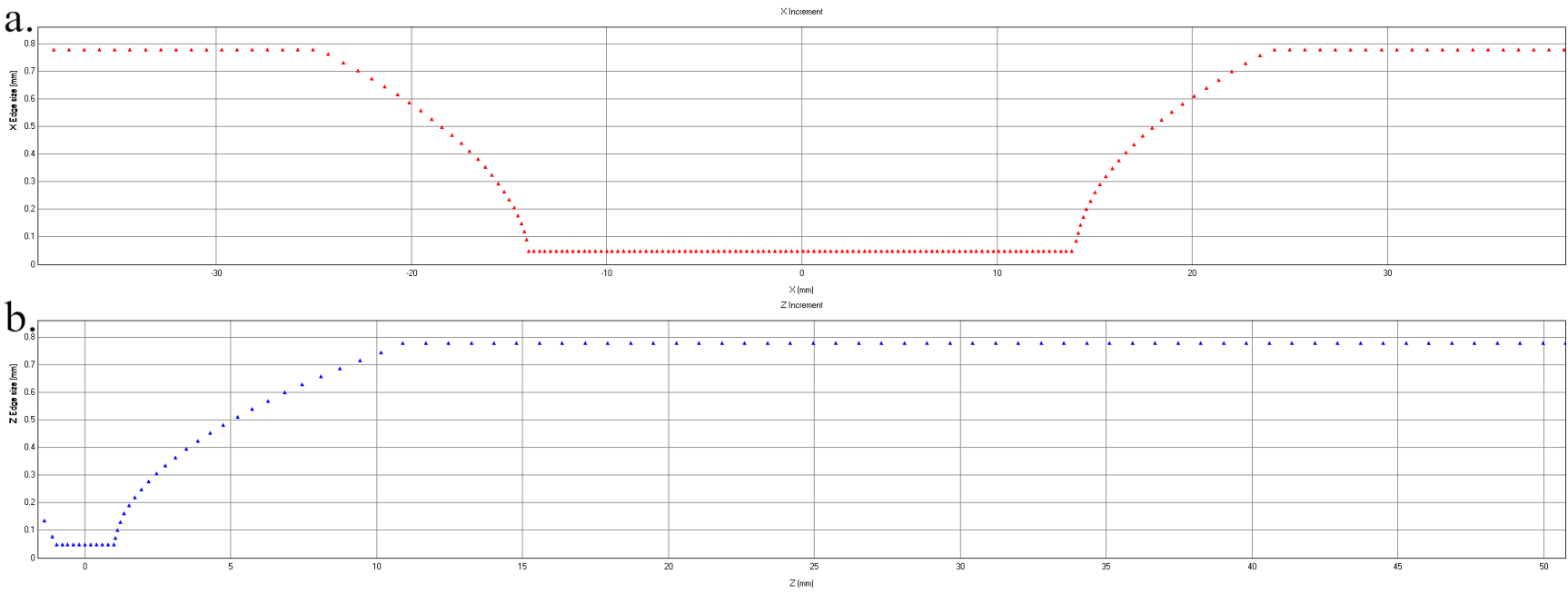

FIG. 3. Adapting mehsing in $\mathrm{x}(\mathrm{a})$ and $\mathrm{z}(\mathrm{b})$. The $\mathrm{y}$ axis is excluded as it is identical to the $\mathrm{x}$. The algorithm uses logarithmic scaling to adjust regional cell sizes.

found via linear interpolation:

$$
\begin{aligned}
& f\left(x, y_{0}\right) \approx \frac{x_{1}-x}{x_{1}-x_{0}} f\left(x_{0}, y_{0}\right)+\frac{x-x_{0}}{x_{1}-x_{0}} f\left(x_{1}, y_{0}\right), \\
& f\left(x, y_{1}\right) \approx \frac{x_{1}-x}{x_{1}-x_{0}} f\left(x_{0}, y_{1}\right)+\frac{x-x_{0}}{x_{1}-x_{0}} f\left(x_{1}, y_{1}\right) .
\end{aligned}
$$

Then the same calculation is run using the newly gotten points but with $y$ :

$$
f(x, y) \approx \frac{y_{1}-y}{y_{1}-y_{0}} f\left(x, y_{0}\right)+\frac{y-y_{0}}{y_{1}-y_{0}} f\left(x, y_{1}\right)
$$

The new field is then summed with other fields at the same point when determining the PIC behaviours.

Two different iterations of the particle in cell program were run. For the first iteration, the macro-particles were 270 microns in each direction forming a rectangle and had a charge of 1 e. The mesh was $198 \times 98$ and the time step was 5ns. As such, this iteration is low density and was more of a test to show the effects of the RF field on the particles. The second iteration had a smaller time step of $0.1 \mathrm{~ns}$. There were 100,000 super-particles each with a charge of $10 \mathrm{ke}$ and a mass corresponding to the charge/mass ratio. An example is shown in figure 4. Each of these simulations assume the chamber of the resonator is full of deuterium gas, and that there are some free electrons within as well.

\section{DISCUSSION}

The outputs of the PIC simulation show two different things depending on the simulation. In the first, the electrons are peeled away in the region with a strong magnetic field, streaming to the sides of the chamber and streaming out of the chamber. In this simulation, the ions remain still. The second simulation shows ion movement, and thus shows waves in the plasma formed. Furthermore, the ions are pulled towards the center, and flow out of the device. This shows an ideal situation in which an ion current out of the device forms regardless of the lack of an extraction electrode. Furthermore, this simulation shows the most movement around the region in which the magnetic field is strongest, indicating a region in which the plasma would likely form.

There are some limitations to this however, due to the difficulty in ionizing deuterium. Deuterium gas would likely need to be accompanied with argon or some catalyst, which was not implemented in the simulation. Fur-

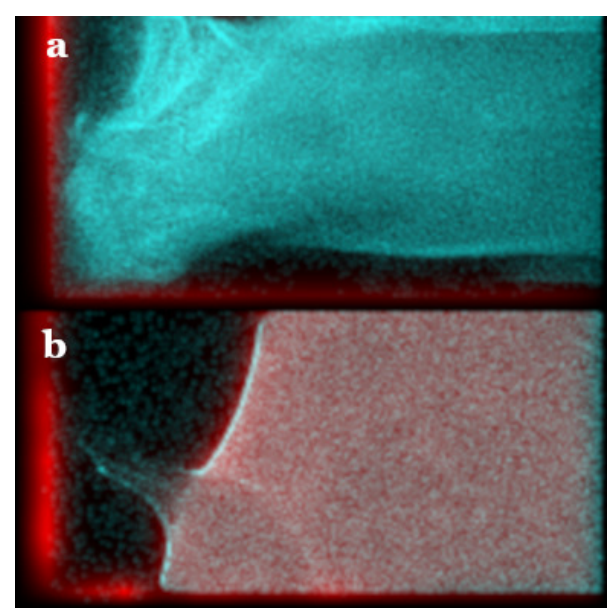

FIG. 4. The 146th iteration in the particle and cell simulation a). the second running of the simulation, showing waves in the plasma and ions flowing out to the right $b$ ). the first simulation showing low movement in ions and removal of electrons from the high magnetic field location. In both, red regions indicate high negative charge and the blue indicates the presence of a particle. 
thermore, the simulation uses the assumption of a uniform gas distribution as opposed to an inlet of deuterium gas. To properly investigate this device, gas needs to flow into the chamber over time.

\section{FUTURE WORK}

As mentioned in the last section, the simulation requires more complex gas composition to model more realistic situations involving deuterium gas. This would involve implementing different reaction cross sections and conditions into the simulation. There should also be analysis involving different gasses to show how this device could be used in situations other than an IEC.

To properly compare the results with common industry standards, an extraction system should be used, and the model should be run with SIMION. The comparison of the results will allow for validation of the simulation. Another comparison which would further validate this is the application of FDTD into the PIC system. While it would likely run slower, developing the fields from FDTD would allow for the plasma to evolve alongside the fields and for the latter to be informed by plasma parameters.

\section{CONCLUSION}

The analysis of an RF electrode was provided to show the integration of FDTD and PIC simulations in order to find outputs for complex plasma sources. To do so, an FDTD simulation developed fields which were interpolated for PIC simulations. Two simulations were run and compared. Finally, limitations and future directions were discussed to show how this blending of methodologies could be improved.

\section{ACKNOWLEDGMENTS}

The author would like to thank Jeffrey Black both for his contributions: the PIC program and his continued guidance and assistance, Christopher Halseth for his RF electrode design which provided an interesting and challenging device to model, and Erik Sánchez for his support, guidance, and design for the resonator body for the ion source.
[1] R. Tanaka, H. Osawa, T. Tabata, T. Ishibashi, and M. Ohnishi, Optimal shape of electrodes for high performance of inertial electrostatic confinement fusion, in $\mathrm{Fu}$ sion Engineering, IEEE/NPSS Symposium on, 20 (IEEE, 2003).

[2] G. H. Miley and S. K. Murali, Inertial Electrostatic Confinement (IEC) Fusion (Springer, 2014).

[3] T. Takamatsu, K. Masuda, T. Kyunai, H. Toku, and K. Yoshikawa, Nucl. Fusion 46, 142 (2005).

[4] T. Burnett, R. Kelley, B. Winiarski, L. Contreras, M. Daly, A. Gholinia, M. G. Burke, and P. J. Withers, Ultramicroscopy 161, 119 (2016).

[5] Y. Takao, H. Koizumi, K. Komurasaki, K. Eriguchi, and K. Ono, Plasma Sources Sci. Technol. 23 (2014).

[6] S. Mattei, M. Ohta, and A. Hatayama, Rf plasma modeling of the linac4 h ion source, in Third International Symposium on Negative Ions, Beams and Sources, 3 (AIP, 2012).

[7] T. Hayamia, S. Yoshinaria, R. Terasakia, A. Hatayamaa, and A. Fukanob, Analysis of discharge initiation in a rf hydrogen negative ion source, in Second International Symposium on Negative Ions, Beams and Sources, 2 (AIP, 2010).

[8] P. J. Ford, S. R. Beeson, H. G. Krompholz, and A. A. Neuber, Phys. Plasmas 19 (2012).

[9] V. P. Gopinath and T. A. Grotjohn, IEEE Trans Plasma Sci 23, 602 (1995).

[10] J. D. Lawson, Some criteria for a power producing thermonuclear reactor, in Proceedings of the Physical Society, Section B, 70 (IOP Publishing, 1957).

[11] S. . K. Murali1, J. F. Santarius, and G. L. Kulcinski, Plasma Sources Sci. Technol. 19, 4 (2010).
[12] P. T. Farnsworth, U.S. Patent No. 325,840,2 (28 Jun. 1966).

[13] B. J. Egle, J. F. Santarius, and G. L. Kulcinski, Fusion Sci. Technol. 52, 1110 (2007).

[14] Y. Gu and G. H. Miley, IEEE Trans Plasma Sci 25, 331 (2000).

[15] S. K. Murali, Diagnostic study of steady state advanced fuel (deuterium-deuterium and deuterium-tritium) fusion in an IEC device, Ph.D. thesis, The University of Wisconsin - Madison (2004).

[16] K. S. Yee, IEEE Trans. Antennas Propag. AP-14, No. 8, 802 (1966).

[17] R. F. Remis, J. Comp. Phys. 163, 249 (2000).

[18] W. H. Press, W. T. Vetterling, S. Teukolsky, and B. P. Flannery, Numerical Recipes in C: the Art of Scientific Computing (Cambridge University Press, 1995).

[19] R. J. Leubbers and F. Hunsberger, IEEE Trans. Antennas Propag. 40, No. 11, 1297 (1992).

[20] A. Taflove, M. Pikey-May, and S. C. Hagness, Fdtdhow complex a problem can we solve?, in Antennas and Propagation Society International Symposium, 4 (IEEE, 2000).

[21] A. Taflove and M. E. Brodwin, IEEE Trans. Microw. Theory Tech. 23, 623 (1975).

[22] G. Mur, IEEE Trans. Electromagn. Compat. 23, 377 (1981).

[23] Z. P. Liao, H. L. Wong, B. P. Yang, and Y. F. Yuan, Scientia Sinica, Series A 27, 1063 (1984).

[24] T. Kashiwa and I. Fukai, Microw. Opt. Technol. Lett. 3, 203 (1990).

[25] R. Luebbers, F. Hunsberger, K. Kunz, R. Standler, and M. Schneider, IEEE Trans. Electromagn. Compat. 32, 222 (1990). 
[26] R. M. Joseph, S. C. Hagness, and A. Taflove, Opt. Lett. 16, 1412 (1991).

[27] V. A. Thomas, M. E. Jones, M. J. Piket-May, A. Taflove, and E. Harrigan, IEEE Microw. Wirel. Compon. Lett. 4, 141 (1994).

[28] J. Berenger, J. Comput. Phys. 114, 185 (1994).
[29] A. A. Vlasov, Sov. Phys. Usp 10, 721 (1968).

[30] A. F. Oskooi, D. Roundy, M. Ibanescu, P. Bermel, J. D. Joannopoulos, and S. G. Johnson, Comput. Phys. Commun. 181, 687 (2010). 